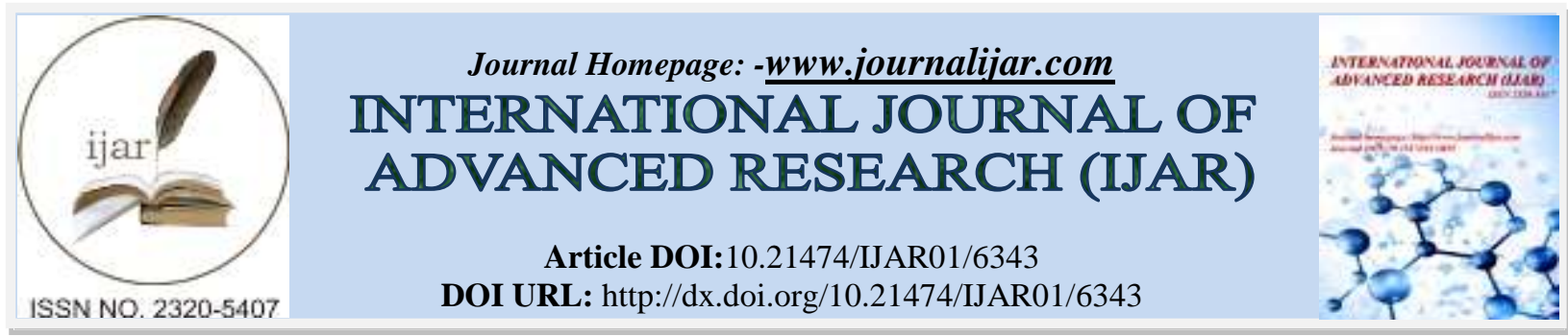

RESEARCH ARTICLE

\title{
SYRIAN REFUGEE CRISIS IN IN JORDAN.
}

Ali Mohammad Hasan Thynat.

Research Scholar in Faculty of Law, Aligarh Muslim University, Aligarh, Uttar Pradesh 202002, India.

\section{Manuscript Info}

\section{Manuscript History}

Received: 18 November 2017

Final Accepted: 20 December 2017

Published: January 2018

\section{Abstract}

The study examines the issue of Syrian refugees in jordan in the field of international relation and the effectiveness of the international community organization and international bodies in resolving this crisis and show the role of intenational community and host country to manage this issue .This study based on the analytical descriptive and approach to review the most important problems related to psychological, family, and economic dimension. In addition, study if there are statistically significant differences in the problems experienced by the Syrian refugees due to demographic variables (gender, number of family members).the results show that the most prominent problems of ther refugees family and the average extent of their failure to benefit from the guidance programs provided to them and on other hand the Economic Disability of the host country to assist Syrian refugees. 


\section{Introduction:-}

Warsarethe primaryandprimary causeofthecrisesanddisasters experiencedbythe peoples. Thesewarscanlastformany yearsandresult ina structural processthatcan be extendedfora longtime.Thesewars haveagreatnegativeimpactthataffectsthefamiliesprimarily because they havetomigrate orresorttosafety places.Theresortneedsacountry that hoststhe next sizeandwithoutwarning.Here,greatproblemsarise from thisresort.Thefamily remainsstrugglingwithitspainsandsorrows andseekstolivewiththereality ofwarinawaythatmay nothaveits components.Securityandsafetyremainthedreamofeveryone.Toachieve atthistimeofwarandmigration, civilianshave beendisplacedeither internally or toothercountries.Neighboringcountries,particularly Lebanon,Turkey,Jordan,IraqandEgypt, openedtheirdoorstothe refugeeswith greatjoy, respondingto that great humanitarian need, and internationalaidagenciesrespondedtothisneed(luck, 2016).

Thelarge numberof refugeeshaschangedthe local demographics, creatingmajorchallengesfortheaffectedcommunity andthehost country'scommunity,affecting the benefit of thesecommunities.A number of these societalchallengesare linkedto educationasanexample andtheneedtoimprovelivelihoodstoreducechildlabororearlymarriage, whichisoneof thereasonsthatnegativelyaffectthehostcommunity as well.Thesizeoftherefugeecommunityisproportionaltothe sizeofthe hostcommunities.However,tensionbetweenhostcommunitiesandSyrian refugeesinsociety hasbecomeevident,andgovernmentandcommunity discourse onrefugeesinthestate has become,inaconcreteway, disquieting.

Syrianfamiliesbegantocometotheterritory oftheKingdom of Jordan throughthe border of Jaberand in legitimateways, because ofthe tribalextensionandsocial,economicand historicalrelationsbetween the inhabitants of theSyrianprovince ofHomsandthepopulation of the JordanianMafraqGovernorate, asis thecase betweenthe Jordanian populationofIrbidandtheSyrianshield.InthefirstsixmonthsoftheSyrianuprising,Jordanianfamilieshostedtheirrelativesa ndrelativesfrom

charitiesacceleratedtoprovideassistance Syrianfamiliesdisplacedbytheevents, whilethecivileffortsofsomelocal residinginJordaniancitieswiththeirmodestefforts, orincooperationwith tothefamily

Syriancitizens distributingaidtoSyrianfamilieshostedin theJordaniangovernorates(UNHCR,2016A).

\section{Importance of Studying:-}

Theimportanceofscientificandappliedstudiesishighlightedinthe following:

TheimportanceofthisstudyistheimpactoftheSyriancrisisonthe situationofrefugees inJordanandafter researchandinvestigationof academicstudieslookingatthesubjectofthecurrentstudytheSyriancrisis andits problemson theSyrianrefugees in Jordan,"theresearcherfaceda lackofpreviousstudiesthathavebeenexposedtotheseproblemsandlack of time The crisisisstillnear andithasnotbeenlonginthe future, sothis studywillenrichtheacademicstudies, andthestudywillenrichthescience byproposingrecommendationsthatcontribute toaddressingthe problems of the Syrianrefugees.

\section{Practical Importance:-}

Thepracticalimportance ofthisstudy willshowtheresults, suggestions andrecommendations ofthe organizationsthat provide servicestotheserefugeesinJordantosolvetheir psychological,family, economic,andguidanceproblemsandhelpthemovercome them.

Objectives of the Study:-

Identifythe socialcharacteristicsof SyrianrefugeesinJordan.

IdentifythepsychologicalproblemsfacingtheSyrianrefugeesin Jordan.

Identifytheproblemsrelatedtothefamilydimensionfacingthe SyrianrefugeesinJordan.

Identifytheproblemsrelatedtotheeconomicdimensionfacedby SyrianrefugeesinJordan.

Identifytheproblemsrelatedtotheextensiondimensionfacedby SyrianrefugeesinJordan. 
Identificationof the relationshipSomespecific gender,numberoffamilymembersandproblemsfacedbySyrianrefugees inJordan.

variables,suchas:

\section{Study Questions:-}

Whatare the characteristicsof SyriansocialrefugeesinJordan?

WhatarethepsychologicalproblemsfacingtheSyrianrefugeesin Jordan?

Whataretheproblemsrelatedtothefamilydimensionfacingthe SyrianrefugeesinJordan?

Whataretheproblemsrelatedtotheeconomicdimensionfacingthe SyrianrefugeesinJordan?

Whataretheproblemsrelatedtothe extensiondimensionfacingthe SyrianrefugeesinJordan?

Aretherestatistically significantdifferencesintheproblems

refugeesduetothegendervariableandthenumberof familymembers?

experiencedby

\section{The Development of the Syrian Crisis:-}

Theworst pessimists did notthink that the demonstrations inDaraawouldturnmonthslater intoabitterwarbetweentheSyrianarmy andthe oppositionfactionsinmostoftheSyrianprovinces,Daraa'sprotests, which cameinlinewiththerevolutionsofanumberofArabcountries, developed

intoviolentconfrontationswithpolice, violenceandmassarrests, resulting inhundreds ofcasualtiesandthousands of injured,However, these measurescouldnotcontain thecrisis that quickly moved to otherSyrian cities.ThecityofHomsandthecountrysideofDamascuswitnessedhuge demonstrationsdispersedby thesecurity

forces,andtherewereanumber of casualties(Watenpaugh,etal,2014)

ByMay, the Syriancrisishadenteredabloodyphaseofthe entryof theSyrianarmy intothecity ofHoms.Thecountry alsowitnessedmore casualtiesinIdlib, RastanandTalbessa.TheSyriancrisiswas characterized byanother split, which resulted in thedissolution of a numberofSyrianarmy officersandtheir establishment ofthe so-called "FreeArmy"ledby dissidentColonelRiadal-Assaad.TheFreeArmy foughtbattleswiththeSyrianarmy intheareasofRastanandHoms, and theFreeSyrianArmyannounceditsfirstattackonamilitaryfacilitysincethe protests began, attackingthe air intelligence headquartersinthe town ofHarastanearDamascus.Inconjunctionwiththebeginningofthearmed conflictinthecountry, theUnitedNations HumanRightsCouncilheldan emergency sessiononDecember2todiscussthesituationinSyria, concludingastatementstrongly condemnedtheviolenceinthecountry, whichwasconsidered toamounttocrimesagainst humanity (Phillips, 2012).

Jordan'sattempttocalm publicdiscontentby narrowingSyrian protectioniswrong. The containmentofthe Syrianrefugee crisisdoesnot addressthe mainstructuralissuesinJordanattheheartofthechallengesit faces, deniesthebenefitsofhostingSyrianrefugees, andignoresthemoral imperative to provideasafehavenfor the victimsofone of theworst humanitariancrisesof this generation. Perhapsmostworrisome isthatthe responseofSyrianrefugeestofurtherdestitution anddespairincreasesthe riskoftherefugeecrisisanditscostsforJordanand the international communityinthe longterm(Ibrahim,2015).

Jordanmustcontinue tobenefitfromtheSyrianrefugee crisisasan opportunity toaddresspreconflictproblems. Theinfluxofrefugees providesJordanwiththe internationalstandingneededtodraw the attentionofinternational donors to thedeep-seated nationalchallengesin the country.However,thisrequiresthe support of the international community.Jordanandinternationaldonorsmustthereforetakeadvantage ofpotential opportunitiesforSyrianrefugeestoenter the Kingdom, promote nationaldevelopmentinJordan, andraise theburdenofSyrians displacedbythe mostdevastatingcivilwarsofthe lastdecade.

\section{Brief Historical Background:-}

TheHashemiteKingdom ofJordanisbarrenandresourcepoor.In thewest, Jordan,sitting onanaridplateau,bordersanarable plot on the banks ofthe JordanRiver,bordering the desertinthe eastand south.As evidence oftheharshnatureofitslandscape,theremainsof theonly ancientcivilizationthatembracesthe JordanValley,ormerelyabandoned defensivesitesdatingbacktothe Romanera.Sinceitisnotnaturaltobea largecountry,Jordan'ssecurity andprosperity dependtoalargeextenton the interestsofforeignpoliticalactorssincetheBritishfirstenvisionedits bordersin1921.Jordan'sgeopoliticalhistory preparedtheKingdom for contemporary challenges.Jordanispoliticallyweakduetoitsresource poverty, its outwardorientedrentereconomy, limited internalrevenue sources, andexcessivepopulationgrowth.Atacrossroadsinaturbulentregion, hehasalongrecordofprovidingasylum 
totheoppressed. Throughoutitshistory,theKingdom hasbeengenerousinusingrefugees toobtaingreaterpolitical and economicsupportfromitssponsors.Indeed, whilethe sizeof theSyrianrefugeecrisisisadifficultchallenge,the Jordaniangovernment'sresponse totheinflux ofSyrianrefugeesmustbe understoodasacontinuationof thehistoricresponsetorefugee flows to thecountry,particularly toPalestinianrefugeesandIraqicommunities. Theincreaseininternationalaidwasaccompaniedbytheinfluxofrefugees intothe Kingdom.Inaddition,Jordan has been usedtoovercome difficultiesinitssurvivalthroughforeignaid,andtheinfluxof refugees hasprovidedopportunitiestobenefitfrom internationalsupport (Rashaida,2014).

TheSyriancrisisaffectingSyrianterritoryanditsinfluenceinmany countriesoftheworldintheEastandWesthasbecomeaproblem Jordaninthematterofasylum.Somemayseethisshiftfromthemainissue tothe for talkinlocalJordaniancirclesshiftsfrom how to resolvethecrisistotalkingaboutsolutionstotherefugeecrisis talkingcalJordaniancirclesshiftsfiom how to resolvethecrisistotalkingaboutsolutionstotherefugeecrisis inJordan. However, thistalktouchesonthe Jordanianreality ina dynamicandno doubtfocusedonitclaimedto producetangibleresultsatthelocallevel, whetherforthebenefitoftherefugeesthemselvesortheinterestofcitizens intheKingdom.Itisthereforenecessary tocontinuetocallupondonor countries to providesupporttotheJordanian governmenttocounter this pressureoneconomicand domesticresources, butthestateneedsto channelthissupporttowardssustainabledevelopmentprojects morethan directrelief.Lawsandregulationsmustbecreatedto supportthe integrationof refugees intothe society,toinvest their abilities,to bring them intothelabormarketandtoproducethem withlegalprotection. Otherwise,asylum willremainaburdenonthenationaleconomyandin needof relief assistance(Chatelard, 2012).

On9/7/2012,the JordanianCouncil ofMinisters decidedupon the recommendationof theMinister ofForeignAffairstoapprovethe establishmentofemergencycampsforSyrianrefugeesintheKingdomand tobeginreceiving Syrianrefugeespresentinthem.Theinternational organizations shouldallow theestablishmentofarefugeecampforthose crossing toJordan fromSyrian territory. ,And decidedto adopt the JordanianHashemiteCharity OrganizationasthesupervisoroftheZa'tari refugeecampinSyria,andthat theUNHCRwillbear thefullexpensesof thecampanditsnecessitiesinaccordancewithanagreementsigned.

Receptiveanddistributionofin-kindandfinancialassistancetorefugees

fromthetwowallsoflocalandinternationalassociations(Al-Lawzi,2013).

The groundworkfor the establishment of the refugeecampwas completedon17/7/2012 withanareaof $7 \mathrm{~km}$. On29/7/2012,theUNHCR FieldCoordinatorannouncedthatZa'taricampwasready toreceive refugeesaccordingtointernationaland humanitarianstandards(Al- Lawzi,2013).

On31/7/2012,thecampwasopenedby theJordanianMinisterof Interior. Thefollowingday, the number ofrefugeeswhowere transferred tothecampfrom thecampsofRamthaandthepeoplewhocrossedthe borderwas 800people.TheZa'taricampislocatedonthelandsofAl ZaatariMunicipality, Thecampisnowthefifthlargestcity inJordanand hasbeenequippedwithbasicinfrastructureandservicessuchaselectricity, waterandroads.Itisequippedwithtentsandprefabhousesthattherefugee needs(Al-Wazzani,2014).

TheHashemiteKingdom ofJordanisoneofthemostrecognized countries intheworld inreceivingwaves ofrefugeesand displaced persons.Ithasreceivedrefugeesanddisplacedpersonsfrom Palestine, Iraq,Syriaandothers.Theflowofasylum toJordanhasdepletedlimited localresourcesand hasputpressure on infrastructureand services.Al- MafraqGovernorate andIrbidGovernorateareamong theworstaffected by theSyrianasylum. Theovercrowdinginthehealthcenters, thehigh demandforwater, the overcrowding of studentsin theschools inthe morningandeveningperiods, andothers. Jordanianscannotaffordtopay thecostofhousing. We shouldnotforgetthatthe lackofgrantsand assistancetotheJordaniangovernment, whichispaiddirectly refugeeswhileburdeningtheburdenofspendingonrefugees.Withannual benefits(Al-Lawzi,2013).

\section{Previous Studies:-}

Thereisnospecializedstudy aimedatthesocial,culturaland economic problems of the presence of Syrianrefugeesat the governorate leveloratthelevelofdifferentregionsinJordan.However,therearemany studiesandresearchesthat dealtwiththe impactofrefugeesonJordan, whichdealtwiththeeconomicandsocialimpactcompletely and comprehensivelyIncludingthemostaffectedprovincesinthe northof the Kingdom,whichabsorbed morethan $50 \%$ oftherefugees, asinthe 
governoratesofMafraqandIrbid.Itisuseful,however,torefertosomeofthestudiesonthesubjectofrefugeeswithsomequantit ativeandobjective analysis, some of whichcanbe referredtoasfollows:

"TheEconomicand Social ImpactofSyrian Refugees onJordan: TheFrameworkoftheSpousesand theMughram," KhaledAl-Wazzani, ConradAdenauer Foundation,Amman,2014.

Thisstudycamewithintheframeworkofanintegrativedealingwith

the positiveandnegativedimensionsoftheSyrianasylum crisisonthe Jordanianeconomy.Forthefirst time, itpresentedan integratedmatrix of macro,sectoralandmicro-effects, whichwouldconstitute costsand revenuesfor theJordanianeconomy.Pointingout that it isnolongera secretthattheJordanianeconomy managedtoachievea set of gains becauseofthe large influx ofrefugees on itsterritory,atthesametime, whichcarrieswithhimmany oftheminesandthelargematerialand community costs.Itwasfairthatthestudyshouldprepareanintegrated balance sheet, alongside the creditor andthe debtor, for the effects of the Syrianrefugeecrisis onthenationaleconomy.Thebalanceof theaudit showedclearly,bothatthemacroandmicrolevels oftheJordanian economy, that the burdenwasmuch greater than the benefits thatthe Jordanianeconomyreceivesduringtheperiod2012-2014.

"EconomicandSocialImpacts of theSyrianCrisisontheJordanian Economy",KhalidAlWazzani,EconomicandSocialCouncil,Amman, 2012.

Thestudyaimedatsheddinglightontheeconomicandsocialimpact oftheSyrianrefugees'flowonJordan,andfocusedmainly onthe direct and indirecteconomic costs onthe nationaleconomy, while takingan analyticallookatsomeofthesocialimpactsofthecrisisontheKingdom's

economy.ThestudydoesnotdealwiththeimpactofthecrisisthatSyria isexperiencingonthenationaleconomy,butismainly confinedtothe economicandsocial impactoftheinflux ofmore than 216,000Syrian refugeesdistributedbetweentherefugeecampsandthegovernoratesofthe Kingdom betweenthebeginningoftherefugeecrisisinMarch2011and the last quarter of 2012,And sheds light onsomefactsandfigures regardingtherealityoftheproblemofSyrianrefugeesinJordanaccording tothe data available tothe UNHCR andthe officialJordaniandata.

Thethirdpartofthe study wasdevotedtotheeconomicandsocial impactof the Syrianrefugeeson the Jordanianeconomy,basedona quantitative analyticalmethodology that takes into consideration theindividual'scostandthefullquantitativeimpactofallrefugees duringthe periodfrom June2011untiltheendofOctober2012.Thestudypointsto

thedirectimpactontheJordanianlabormarketandtheacquisitionofabout38,000jobsbytheSyrianrefugeesuntil2012, about 40\%oftheannualemploymentopportunitiesforJordanian labor, whichisanimportant challengetothenationalemploymentstrategy ofnationalemployment policiesinthecountry.

AbuTarboush,(2014), entitled"TheSocialandPsychological Effectsof theSyrianCrisisonSyrianRefugeeChildreninJordan", which

aimedtoidentifythemostimportantsocialandpsychologicaleffectsofthe Syriancrisisonthe Syrianrefugee childrenin Jordan;the relationship betweentheseeffectsby genderandage,Theexistenceofthechildin Jordan,and theextent towhichitrelatestotheproblemsfaced in the countryofasylum. Thequestionnairewasusedbyanumberofassociations andnon-governmentalorganizationsin Jordan, which providesocial supportprogramsfor childrenandtheir parents. The sample wasused asa study toolforeachof the twosub-samples(childrenandparents)Butthe majority weremoderate.Thehighertheage, thelowerthe levelof psychosocialeffects, andtherearenodifferencesinthesocialand psychologicaleffectsof psychosocialeffectsonchildreninvarying degrees(low, highaverage).

Study of"PalestinianRefugeesfrom SyriatoLebanonLegaland $\quad$ HumanChallenges," PalestinianHumanRightsFoundation(SHAHID), FieldStudy,September,(2012).

Ateam from thePalestinianHumanRightsFoundation(SHRAD) conductedafieldvisittoPalestinianrefugeesfrom Syriaandresidentsof the PalestiniancampsintheLebaneseRepublic(North,Bekaa,Beirut, SaidaandTire)andrecordedthereality,circumstancesand needs ofthe refugeesinaspecialform preparedforthispurpose.(1837)Palestinian familiesfromSyriatoLebanonuntilSeptember(2012).Thestudy concludedbylookingatthefiguresofthepopularcommitteesandcharities throughLebanesepublicsecurity estimates.Thestudy aimsatidentifying theneedsofourrefugeesfrom SyriatoLebanonandidentifyingareasof 
interventiontoassistthem andraisethemtotheinternationalcommunity fordonorsandinternational,regionaland localorganizationsin orderto encourage themto help them. The studyfound a number of results, includingthattherefugeefamilieshavebecomeanadditional burdenon thechildrenofthecamps,especially thattheexpatriatesaremostly livingwiththeirrelativesin smallhouses, forcingsomemembersofPalestinian familiestoleavetheirhomes. Theeconomicconditionsofthehostfamilies areaggravatedby theabsenceofservicesforthenewresidents. Thereare healthriskstotherefugeepopulationbecausethey arenotcoveredby the fullhealthcareprovidedby UNRWAtoregisteredPalestinerefugees. Theserisksarevery highforchildren.Thereisnoclearvisionsofarfor UNRWAbecausethecurriculadifferbetweenLebanonandSyriaandlarge numbersof studentsmay notbeaccommodated.Non-governmental Palestinianorganizationshavefoundthemselvesfacinga haveshiftedtheirattentiontorefugeesattheexpense oftheirtarget groups. majorchallenge;

they

Study of theImpactoftheSyrianCrisisonJordan,Ministry of PlanningandInternationalCooperation, Jordan, 2014.The report tackled the impact of the Syriancrisis onJordan in generalandfocusedon the impactofSyrianrefugeesonJordaniansocietyinseveralareas:education, energy, health,housing,employment,unemployment,socialprotection andwater.According tothereport,Mafraqgovernoratewas one of the mostaffectedbytheSyrianrefugees'emigration.Theunemploymentrate amongJordanianyouthincreasedduetotheemploymentofSyrianworkers insteadofJordaniansbecauseofthelow wagesofSyrian workers.In addition, thereis an increasein thepercentageof child laborin the JordanianmarketfollowingthedisplacementofalargenumberofSyrians toJordan. Thereportpointedoutthatthereisanincreaseindemandin some areasinJordanfor goodsamountedto $300 \%$.

"TheImpactofRefugeesontheEconomy oftheHostCountry, The CaseStudy ofJordan,intheNameofAlLuzi,"(2013)InterdisciplinaryJournalof ContemporaryResearchinBusiness.

Thestudy examinedtheimpactofrefugeesontheJordanian economy.Theimpactwasmeasuredby the unemploymentrate, direct investmentsofrefugeesandhighprices.Thestudy foundthatthereisa positive effecton the unemployment and food prices of refugees.In addition,there is asignificant impacton the politicalsituation ofrefugees inJordan,asindicatedbythestudysamplewhichreached65\%, while 70\% Thesocialaspectand60\% thestudiedsamplereportedthatthere isan environmentalimpactof refugees.

Study ofthe"ImplicationsandCostsofHostingIraqiRefugeesin Jordan",NorwegianFafoFoundation,2007.Thisstudy comesafterFafo finishedthefirstsurveyatthelevelofneighboringcountriestodeterminethe numberofIraqis, theirplaces, theirsocialclasses, andtheir sectarian andethnicbackgrounds. The survey revealedthatthenumberofIraqisin Jordanisestimatedat(450)thousand, aswellas50thousandmoversinside and outside the Kingdom, while officialestimates indicate there are (750) thousandIraqisinJordan.

Theresultsofthestudy

revealedthehigheducationofthemajority ofIraqisresidingintheKingdom.HalfofIraqisareovertheageof 25,28\% areunder 15, andtherestarebetween 15and25yearso ld.Insectarian andethnicdistributions, thesurveyshowedthateightoutofevery10Iraqis followtheSunnifaithor areof Araborigin.

\section{Method of the Study:-}

Thisstudy isbasedontheanalyticaldescriptivedescriptive approach.Thismethodwas used toreview themostimportantpractical fieldstudiesrelatedtothe problemsfacedbySyrianrefugees.

\section{Study Society:-}

Thestudypopulationconsistsofmorethan(20,000)Syrianrefugees familyinthe refugeecampinjordan(UNHCR,2016).

\section{The Study Sample:-}

Thesampleofthestudywasselectedintheavailableway(available) duetothedifficultyofreachinganorganizedrandomsampleoftheSyrian families, duetothe lackof organizedrecordsof thenamesofthese families.Thiswasdoneby interviewingtheorganizationsworkingwith the Syrianrefugeesinthe camp's, Withtheassistance of socialworkers workinginthoseorganizations; thetotalnumberoffamilieswasmorethan $(20,000)$. 
Afterthequestionnairewasretrieved, 10questionnaireswere excludedbecause they werenotvalidfor statisticalanalysis, becausethe datawerenotcompletedorduetotheinaccuracyofthedatabecausesome respondentsdidnot cooperateorabstained, Thefinalsamplewas(110) questionnaires.

\section{Questionnaire Study Tool:-}

The studytoolconsistedofthestudyquestionsandconsistedof:

Section 1:-Demographic information: gender,age,maritalstatus, familystatus,numberoffamilymembers,educationalstatusoftheheadof household(husband), educationalstatus of the head of household,current headofhousehold,monthlyincomeofthefamily.Aswellassomegeneral informationcovering thenature of socialwelfare servicesprovidedto refugees.

Thesecondsection, whichmeasuresthe study questionsrelatedto theproblemsfacingthe Syrianrefugeesincamp,inthefollowingaxes:

First:the problemsofSyrianrefugeesrelatedtothe psychological dimension,areinparagraphs(02-1)

Second:theproblemsofSyrianrefugeesrelatedtothefamily dimension, areinparagraphs(02-1)

Third:TheproblemsoftheSyrianrefugeesrelatedtotheeconomic dimensionare representedinparagraphs $(02-1)$

Fourth:The problemsofSyrianrefugeesrelatedtotheextension dimensionare representedinparagraphs(02-1)

Validity and Reliability Study Contenttool:-

Thequestionnairewaspresentedtosome offaculty membersof the University's inJordan, andsome specialistsinsocialwork, sociology, measurement, evaluationandstatisticstoverify the veracity oftheir paragraphs.Fromtheobservationsofthearbitrators, by deletion, addition ormodificationuntilthestudy toolappearedinitsfinalform dividedinto three sections. Theresearcherconsideredtheviewsof thearbitratorsand theiramendmentsasanindicationofthevalidityofthecontentofthestudy toolThestudyandtheappropriatenessofitsparagraphsandtheirdiversity, andaftermakingtherequiredmodifications,achieveabalancebetweenthe contentsof the studytoolinitsparagraphs, whichconfirmsthevalidityof thescale.

\section{Stability of the Study Instrument:-}

Todeterminetheconsistencyofeachparagraphofthequestionnaire withtheaxistowhichthe paragraphbelongs, thecorrelationcoefficients betweeneachparagraphofthequestionnairewereusedbytheuseoftheAlphaCronbachcoefficient.Table 1showstheresults of the test; Cronbach'salphavaluesforthestudy instrumentweregenerally higher (0.60), whichisacceptableinresearchandhumanitarianstudiesasinTable

(1):

Table 1:-Resultsof stabilitycoefficientsusingthe Alpha Cronbachcoefficient

\begin{tabular}{|l|c|c|}
\hline Studyvariables & Paragraphs & $\begin{array}{c}\text { Stabilitycoefficient usingthe } \\
\text { Alphacronbach test }\end{array}$ \\
\hline $\begin{array}{l}\text { problems ofSyrianrefugees } \\
\text { relatedtothepsychological dimension }\end{array}$ & $1-20$ & 0.66 \\
\hline $\begin{array}{l}\text { ProblemsofSyrianrefugees } \\
\text { relatedtothefamily dimension }\end{array}$ & $1-20$ & 0.67 \\
\hline $\begin{array}{l}\text { Problemsof Syrianrefugees' } \\
\text { relatedtotheeconomicdimension }\end{array}$ & $1-20$ & 0.64 \\
\hline $\begin{array}{l}\text { ProblemsofSyrianrefugees } \\
\text { relatedtotheextensiondimension }\end{array}$ & $1-20$ & 0.93 \\
\hline Issuesasawhole & 80 & 0.78 \\
\hline Proposalsasawhole & 20 & 0.71 \\
\hline
\end{tabular}

\section{Statistical Processing:-}

In order toanswer the questions of the study, descriptive and analyticalstatisticalmethodswereusedby usingthefrequencyand percentageofthesample ofthesample. Thestatisticalpackagewas calculatedaccording tothedemographicvariables. TheCronbachAlpha testwasusedtoconfirm thestability ofthestudy toolandtoanswerthe questionsThestudyusedthearithmeticalaverages, thestandarddeviations tomeasurethe dispersionof responsesfrom thearithmeticmean, The use One WayANOVA, andScheffeTest. 


\section{Results of the Study:-}

ResultsonfirstQuestion1:Whatarethedemographiccharacteristics of Syrianrefugees?Table (2) Distributionofthe sample of thestudyaccordingtothe demographic data

\begin{tabular}{|c|c|c|}
\hline Variable & Repetition & percentage \\
\hline \multicolumn{3}{|c|}{ Gender } \\
\hline Male & 20 & $6 . .8$ \\
\hline female & 16 & 18.1 \\
\hline Total & 112 & 122.2 \\
\hline \multicolumn{3}{|c|}{ Age } \\
\hline
\end{tabular}

\begin{tabular}{|c|c|c|}
\hline Less than30years & .0 & 02.1 \\
\hline Less than35years & .8 & .0 .3 \\
\hline 35 to less than 40 years & 01 & 12.1 \\
\hline 40-Less than 45 & 11 & 10.3 \\
\hline Less than50years & 5 & 1.5 \\
\hline 50years and over & 0 & 1.6 \\
\hline Total & 112 & 122.2 \\
\hline \multicolumn{3}{|c|}{ Marital status } \\
\hline Married & 21 & 65.5 \\
\hline absolute & 2 & 6.0 \\
\hline Widowed & 3 & 8.1 \\
\hline Total & 112 & 122.2 \\
\hline \multicolumn{3}{|c|}{ Familystatus } \\
\hline Integratedfamily & 21 & 6525 \\
\hline Deceased father & 3 & 8.1 \\
\hline Themother is dead & 1 & .2 \\
\hline Separate & 6 & $3 .$. \\
\hline Total & 112 & 122.2 \\
\hline \multicolumn{3}{|c|}{ numberof familymembers } \\
\hline Less than 3 individuals & 15 & $1 . .8$ \\
\hline $\begin{array}{l}3 \text {-to less than } 6 \\
\text { individuals }\end{array}$ & 8. & $53 .$. \\
\hline 6 -Less than 9 individuals & 06 & 05.5 \\
\hline 9 individuals and more & 1 & .8 \\
\hline Total & 112 & 122.2 \\
\hline \multicolumn{3}{|c|}{ Educational status ofthehead of thefamily(husband) } \\
\hline Illiterate & $\ldots$ & .2 .2 \\
\hline Reads and writes & 01 & 12.1 \\
\hline primary & 02 & 16.0 \\
\hline preparatory & .1 & .2 .2 \\
\hline secondary & 0 & 1.6 \\
\hline Total & 112 & 122.2 \\
\hline \multicolumn{3}{|c|}{ Educational status of thehead of thefamily(WIFE) } \\
\hline Illiterate & 52 & 15.5 \\
\hline Reads and writes & 00 & 02.0 \\
\hline primary & 16 & 18.1 \\
\hline preparatory & 12 & $13 .$. \\
\hline secondary & 1 & .2 \\
\hline Total & 112 & 122.2 \\
\hline \multicolumn{3}{|c|}{ Profession ofthe head ofthe family } \\
\hline private sectoremployee & . & 0.3 \\
\hline freebusiness & 12 & 2.1 \\
\hline Daylaborer & .2 & $03 .$. \\
\hline
\end{tabular}




\begin{tabular}{|l|l|l|}
\hline does not work & 83 & 82.2 \\
\hline \multicolumn{1}{|c|}{ Total } & 112 & 122.2 \\
\hline Monthlyincomeof thefamily & 8. & $53 .$. \\
\hline Less than100 dinars
\end{tabular}

\begin{tabular}{|c|c|c|}
\hline $\begin{array}{c}100 \text {-to less than 150 } \\
\text { dinars }\end{array}$ & 01 & 12.1 \\
\hline 150 -Less than 200 dinars & 02 & 16.0 \\
\hline 200 -less than 250 dinars & 0 & 1.6 \\
\hline 250 dinars and more & 1 &. .8 \\
\hline Total & 112 & 122.2 \\
\hline \multicolumn{2}{|c|}{ Length of stayin the camp } & 6.0 \\
\hline $\begin{array}{c}\text { less than oneyear } \\
\begin{array}{c}\text { oneyear to less thanayear } \\
\text { and ahalf }\end{array}\end{array}$ & 1.6 \\
\hline $\begin{array}{c}\text { one and ahalf to less than } \\
\text { twoyears }\end{array}$ & 0 & 12.2 \\
\hline $\begin{array}{c}\text { twoyears to less than two } \\
\text { and ahalfyears }\end{array}$ & 11 & 11.6 \\
\hline threeyears and more & 1. & 86.0 \\
\hline Total & 35 & 122.2 \\
\hline
\end{tabular}

Table(2)showstherelativedistributionofrespondentsaccordingtogendervariables:

age,maritalstatus,familystatus,numberoffamily members,educationalstatusof theheadofhousehold(husband), headof household,(83.6\%)oftheheadsoffamily, whilethepercentageoffemale headswas(16.4\%.( Intermsof age,the percentage of youngpeople inthe sampleis high:32.7\% of the totalrespondents, and30.1\% ofthe respondents(aged3035)Oftheagegroup(from 35yearstolessthan 40 years)was $19.1 \%$, andthoseaged $40-45$ yearswerelessthan $45 \%(12.7 \%$ ).

Lessthan50\%(4.2\%)and50\%andabove(1.8\%), indicatinga high percentageRefugee youthareheadsoffamily (30lessthan 35) dueto theirattempttokeeptheirfamiliesfromthedangeroftheSyriancrisisand itsrepercussions. The distributionofrespondentsaccordingto thesocial situation shows that the largest proportion ofthe samplewasmarried $(85.5 \%)$ of thefamily, while (8.2\%)number ofdivorceesofthem.

\section{What are the Problem Sfacing the Syrian Refugees?}

Table (3) Thestatisticalaveragesandthestandarddeviationsofthe responsesof thesample membersofthe studyonthe mostimportantproblemsfacingthe Syrianrefugees.

\begin{tabular}{|l|c|c|c|l|}
\hline Problems & Arithmetic average & standard deviation & Rank & Level \\
\hline Economicproblems & 0.51 & 2.15 & 1 & High \\
\hline $\begin{array}{c}\text { Psychological } \\
\text { problems }\end{array}$ & 0.22 & 2.08 & & Average \\
\hline Familyproblems & 0.23 & 2.06 & - & Average \\
\hline Indicativeproblems & 1.22 & 2.16 & 1 & Average \\
\hline Problems in general (3) the & 2.04 & 2.18 & 5 & Average \\
\hline
\end{tabular}

Syrianrefugees)rangedfrom 2.51 to 1.90 , withproblemsgenerallyhaving

2.14average, andeconomicproblems(2.09), with astandard deviation(0.26)from themiddlelevel,and inthirdplacecamethefamily problemswithanarithmeticalaverage((2)). 2.07), standarddeviation (0.28),middle level, andfourthandbrotherly Theindicative problems camewithanaverage of 1.90anda standarddeviationof 0.48 .This indicatesthatthe refugeessuffer fromvery poor economic conditions.

Resultsrelated tothe second question:Whatare the problems related tothepsychologicaldimension facingthe Syrian refugees?

Table (4) averagesandstandarddeviationsof the answerstothe studysample paragraphsaboutthe axis"problemsrelatedtothe psychologicaldimensionof facingSyrianrefugees" arrangedindescending 


\begin{tabular}{|c|l|l|l|l|l|}
\hline N\# & Paragraph & $\begin{array}{l}\text { Arithmetic } \\
\text { average }\end{array}$ & $\begin{array}{l}\text { Standard } \\
\text { deviation }\end{array}$ & Ranking & Level \\
\hline 02 & $\begin{array}{l}\text { I am worriedabout mychildren } \\
\text { and i mayfacedifficult situations }\end{array}$ & 0.22 & $2 . .2$ & 1 & High \\
\hline 15 & $\begin{array}{l}\text { Isuffer from neglect and } \\
\text { marginalization bythe } \\
\text { international community }\end{array}$ & 0.65 & 2.15 & 0 & High \\
\hline 11 & $\begin{array}{l}\text { Iliveinastate ofinstabilityand a } \\
\text { constant threat of departure }\end{array}$ & 0.60 & 2.58 & - & High \\
\hline 12 & $\begin{array}{l}\text { Ihavethefull courageto face } \\
\text { different situations }\end{array}$ & 0.35 & 2.82 & 1 & High \\
\hline 5 & $\begin{array}{l}\text { Ihavethecourageto facedifficult } \\
\text { situations }\end{array}$ & 0.88 & 2.82 & 5 & High \\
\hline 12 & $\begin{array}{l}\text { Isuffer from depressionbecause } \\
\text { ofthe situation welivein }\end{array}$ & 0.58 & 2.32 & 8 & High \\
\hline 1 & Ilose mynerveseasily & 0.5. & 2.61 & 3 & High \\
\hline 3 & $\begin{array}{l}\text { Ihaveaconstant feeling of } \\
\text { nervousness }\end{array}$ & 0.16 & 2.6. & 6 & High \\
\hline 18 & $\begin{array}{l}\text { Thereis no oneto talk toabout } \\
\text { the problems that arefacingme }\end{array}$ & 0.10 & 2.3. & 2 & High \\
\hline 6 & $\begin{array}{l}\text { Myfeelingof insecuritymakes } \\
\text { me feel veryscared }\end{array}$ & 0.12 & 2.60 & 12 & High \\
\hline 16 & Ican notact freelyin thecamp & 0.15 & 2.2. & 11 & Average \\
\hline- & $\begin{array}{l}\text { Ihavecases ofcryingafter being } \\
\text { in the camp }\end{array}$ & 1.22 & 2.66 & 10 & Average \\
\hline 0 & Negativethoughts cometo mind & 1.38 & 2.28 & 1. & Average \\
\hline 10 & $\begin{array}{l}\text { Ifeel fits ofangeraccompanied } \\
\text { byaggressivebehavior }\end{array}$ & $\begin{array}{l}\text { Ifeel that thejordaniansocietyis } \\
\text { lookingnegativelyat ourchildren }\end{array}$ & 2.22 & 11 & Average \\
\hline 1. & 2.86 & 15 & Low \\
\hline
\end{tabular}

\begin{tabular}{|c|l|l|l|c|l|}
\hline 1 & $\begin{array}{l}\text { Ihesitate to establish relationships } \\
\text { with others }\end{array}$ & 1.11 & 2.3. & 18 & Low \\
\hline 2 & Ifeel thelack ofaid & $1 . .2$ & 2.88 & 13 & Low \\
\hline 11 & Itryto stayawayfrom others & 1.00 & 2.52 & 16 & Low \\
\hline 8 & $\begin{array}{l}\text { I am ashamed of theliving } \\
\text { conditions inside the camp }\end{array}$ & 1.01 & 2.5. & 12 & Low \\
\hline 13 & $\begin{array}{l}\text { Ifeelashamed when dealingwith } \\
\text { others }\end{array}$ & 1.11 & 2.16 & 02 & Low \\
\hline & General arithmetic mean & 0.22 & 2.08 & & Average \\
\hline
\end{tabular}

It is clear from Table (4) that the mathematical averages of psychologicalproblemsfacingtheSyrianrefugeesrangedfrom(2.90to1.14). Theaxisobtainedanaverage of(2.09)(20)at thehighest level of myaccount,itreached(2.90), andthestandarddeviation(0.30), whichis highlevel,theparagraphstated("worriedaboutmy childrenmay face difficultsituations), andthisistheconcernoftheheadofthefamilyforhis childrentoengageIn thequarrelsandthe increase oftheriots, andinthe second placewas paragraph(15)withanaverage of(2.85)and astandard deviation(0.45), which isThisparagraphshowsthatthe international community isnotinterestedinitscauseasarefugee, despiteallthe assistance providedby theinternationalcommunity,anditseemsclear from thenextparagraphthatIliveunderInstabilityAndaconstantthreat ofdeparturewithanaverageof(2.82).This leadstothe feeling of depressionoftherefugeebecauseoftheconditionsheliveswithanaverage of(2).Incontrast, inthe last paragraphcamethenumber(17)withan averageof(1.14) andwithastandarddeviationof(0.48).Iamashamedto dealwithothers.This indicatesthata refugeehasa strongpersonalityand hastheability todealwithothersnaturallyandthattheasylumcrisishas notaffectedhisrelationswithothers. 
Resultsrelated tothe third question: what are the problems related to the family dimension facing the Syrian refugees?

Table (5):- averagesandstandarddeviationsof the responsesof the studysample paragraphsaboutthe axis"problemsrelatedtothefamilydimensionfacingSyrianrefugees" indescendingorder.

\begin{tabular}{|c|c|l|l|l|l|}
\hline N\# & Paragraph & $\begin{array}{c}\text { Arithmetic } \\
\text { average }\end{array}$ & $\begin{array}{l}\text { Standard } \\
\text { deviation }\end{array}$ & Ranking & Level \\
\hline 5 & el unableto meetthebasic needsofmychildren. & 0.62 & $2 . .1$ & 1 & high \\
\hline 6 & $\begin{array}{c}\text { I feel likerunningawaybecause of } \\
\text { a lotofproblems }\end{array}$ & 0.62 & 2.11 & 0 & high \\
\hline
\end{tabular}

\begin{tabular}{|c|c|c|c|c|c|}
\hline 13 & $\begin{array}{l}\text { I amconcernedaboutmychildren } \\
\text { for their education }\end{array}$ & 0.6 . & 2.15 & . & high \\
\hline 15 & $\begin{array}{l}\text { I havealotof problemswithmy } \\
\text { familyonverysmall grounds. }\end{array}$ & 0.33 & 2.82 & 1 & high \\
\hline 18 & $\begin{array}{l}\text { I findthatusing yellingmaywork } \\
\text { formychildren }\end{array}$ & 0.50 & 2.38 & 5 & high \\
\hline & $\begin{array}{c}\text { I usebeatingsas amethodto } \\
\text { disciplinemychildrenwhenthey } \\
\text { makemistakes. }\end{array}$ & 0.11 & 2.38 & 8 & high \\
\hline 8 & $\begin{array}{l}\text { Mychildren donotfollowmy } \\
\text { directionseasily. }\end{array}$ & $0 . .5$ & 2.32 & 3 & high \\
\hline 12 & $\begin{array}{l}\text { I havedifficultyin beingableto } \\
\text { directmychildren. }\end{array}$ & $0 . .1$ & 2.85 & 6 & high \\
\hline 10 & $\begin{array}{l}\text { I findthatconsultationwithmy } \\
\text { children tomakedecisionsis futile. }\end{array}$ & 1.23 & 2.21 & 2 & Average \\
\hline 2 & $\begin{array}{l}\text { Thebestgirlmarriageforminors } \\
\text { when theycamea suitable opportunityfor } \\
\text { them. }\end{array}$ & 1.62 & 2.2 . & 12 & Average \\
\hline 1. & $\begin{array}{l}\text { Mychildren's behavior atschoolis } \\
\text { good. . }\end{array}$ & 1.33 & 2.61 & 11 & Average \\
\hline 3 & $\begin{array}{l}\text { I feelthe crueltyofmychildren } \\
\text { andtheuseofviolencewhen dealingwithothers. }\end{array}$ & 1.38 & 2.60 & 10 & Average \\
\hline 1 & $\begin{array}{l}\text { I feel unabletosolvetheproblems } \\
\text { mychildren face. }\end{array}$ & 1.32 & 2.33 & 1. & Average \\
\hline 12 & $\begin{array}{l}\text { Mybadcircumstances led to the } \\
\text { denial ofmychildren fromschool }\end{array}$ & 1.86 & 2.33 & 11 & Average \\
\hline 02 & $\begin{array}{l}\text { I amthe causeof theproblems } \\
\text { that happen tomyfamily. }\end{array}$ & 1.38 & 2.63 & 15 & Average \\
\hline 11 & $\begin{array}{l}\text { I feelweakin thefaceofproblems } \\
\text { when I thinkmyfamilycanhavea problem }\end{array}$ & 1.85 & 2.21 & 18 & Low \\
\hline 1 & $\begin{array}{l}\text { Mychildren avoidtalkingtome } \\
\text { abouttheirforeignrelations. }\end{array}$ & 1.81 & 2.61 & 13 & Low \\
\hline 11 & $\begin{array}{l}\text { I noticethatthebehaviorof my } \\
\text { children is changingveryquickly towards } \\
\text { thenegative. }\end{array}$ & 1.80 & 2.32 & 16 & Low \\
\hline 0 & $\begin{array}{l}\text { I can notact veryfreely with } \\
\text { regardtofamilydecisions. }\end{array}$ & 1.52 & 2.62 & 12 & Low \\
\hline 16 & $\begin{array}{l}\text { I amnotknown amongmany } \\
\text { people. }\end{array}$ & 1.18 & 2.3 . & 02 & Low \\
\hline & General arithmeticmean & 0.23 & 2.0 . & & Average \\
\hline
\end{tabular}

table(5) showsthatthe mathematicalaverages of (problemsrelated tothefamily dimensionfacingSyrianrefugees (2.89and1.46).Theaxis obtainedan average of(2.07), which istheaverage level,and obtained(5,

80)and(44),respectively, Withastandarddeviationof0.34fromthehigh

level.Thetwoparagraphsstated:"IfeelthatIamunabletomeetthebasicneedsofmychildren.Ifeelthedesiretoescapefromreali 
tybecauseofthe many problems."Thisindicatesthattherefugeesuffersfrommany problemswithinthecamp,Because of the inability of therefugee tomeet the basicneedsof thechildren,andinthe secondparagraph(17)withan averageof(2.83)standard(0.45)ofa high level;asstated onparagraph(I amconcerned) towardsmychildrenfor, althoughthe provisionof schools for the children ofSyrianrefugees, but therefugeesareconcernedabout the future oftheeducationoftheirchildren.Ontheother hand, inthe last paragraph,paragraph(18)camewithanaverage of(1.46) andastandard deviation(0.73).Itisoflowlevel.Theparagraphstates:"Iam notknown amongmany people.Therefugeesinside thecamparestrong, andthey knoweachother verymuch.

\section{Resultsrelated tothe fourth question:What are the problems related totheeconomic dimension facing the Syrian refugees?}

Table (6) The arithmeticalaveragesandthe standarddeviationsof the responsesof the sample membersof the studyonthe issuesof the axisof "Problemsrelatedtothe economic dimensionfacingthe Syrian refugees" rankedindescendingorder.

\begin{tabular}{|c|c|c|c|c|c|}
\hline $\mathrm{N} \#$ & Paragraph & $\begin{array}{l}\text { Arithmetic } \\
\text { average }\end{array}$ & $\begin{array}{l}\text { Standard } \\
\text { deviation }\end{array}$ & Ranking & Level \\
\hline 9 & Householdexpenses morethan income. & 0033 & 3033 & 1 & high \\
\hline 13 & $\begin{array}{l}\text { Oureconomicsituationinsidethe } \\
\text { campneeds greatsupport. }\end{array}$ & 0033 & 3033 & 2 & high \\
\hline 1 & $\begin{array}{l}\text { Economicconditionsmakethe } \\
\text { futureofmyfamilyunstable. }\end{array}$ & 2092 & 3019 & $\mathbf{0}$ & high \\
\hline 11 & $\begin{array}{l}\text { I haveagreatdifficultyin providing } \\
\text { myfamily. }\end{array}$ & 2092 & 3019 & 1 & high \\
\hline 7 & $\begin{array}{l}\text { Mymultipleneedslimitmyaccess } \\
\text { toallmaterialservices. }\end{array}$ & 2097 & 3021 & 5 & high \\
\hline 17 & $\begin{array}{l}\text { Mylackof incomemadethe } \\
\text { availabilityofall fooddifficult. }\end{array}$ & 2092 & 3027 & 2 & high \\
\hline 12 & $\begin{array}{l}\text { Ourcurrenteconomicsituation } \\
\text { insidethe campisnotstable. }\end{array}$ & 2091 & 3001 & 7 & High \\
\hline 2 & $\begin{array}{l}\text { Myphysical conditionlimits my } \\
\text { personalambitions towards abetter } \\
\text { tomorrow. }\end{array}$ & 2022 & 3009 & 2 & High \\
\hline 1 & $\begin{array}{l}\text { Thephysicalsituationof thefamily } \\
\text { is anobstacletoachievingmy children's } \\
\text { educationaspirations. }\end{array}$ & 2021 & 3012 & 9 & High \\
\hline 15 & $\begin{array}{l}\text { I havedifficultypaying mychildren's } \\
\text { tuition. }\end{array}$ & 2075 & 3051 & 13 & High \\
\hline $\mathbf{0}$ & $\begin{array}{l}\text { I thinka lotabout mychildren's } \\
\text { economic futureandI feel likeI'm } \\
\text { notoptimistic. }\end{array}$ & 2027 & 3052 & 11 & High \\
\hline 12 & $\begin{array}{l}\text { Thereareweaknessesin thequality } \\
\text { of servicesandcivil society } \\
\text { institutionstohelpus. }\end{array}$ & 2073 & 3057 & 12 & High \\
\hline 11 & $\begin{array}{l}\text { I tendtorelyoninstitutions thatcan } \\
\text { provideassistance }\end{array}$ & 2052 & 3023 & 10 & High \\
\hline 2 & $\begin{array}{l}\text { That thejobsavailableinthecamp } \\
\text { are limitedandthereforewillnot } \\
\text { makeaneffort togetabetter future }\end{array}$ & 2001 & 3022 & 11 & High \\
\hline 12 & $\begin{array}{l}\text { I haveahardtimeknowingwhich } \\
\text { institutionscan provideassistance }\end{array}$ & 2003 & 3029 & 15 & Average \\
\hline 5 & $\begin{array}{l}\text { Justthinkingofmyfamilybeinghit } \\
\text { byanyfuturefinancial predicament } \\
\text { makesmefeelweakinmyabilityto } \\
\text { faceproblems }\end{array}$ & 2017 & 3092 & 12 & Average \\
\hline
\end{tabular}




\begin{tabular}{|l|l|l|c|c|l|}
\hline $\mathbf{2}$ & $\begin{array}{l}\text { Thepresenceof someunemployed } \\
\text { children (unemployment) }\end{array}$ & $\mathbf{1 0 2 2}$ & $\mathbf{3 0 9 2}$ & $\mathbf{1 7}$ & Average \\
\hline $\mathbf{1 0}$ & $\begin{array}{l}\text { Thelowstandardoflivinghas been } \\
\text { reflected inmyhealth }\end{array}$ & $\mathbf{1 0 7 2}$ & $\mathbf{3 0 9 3}$ & $\mathbf{1 2}$ & Average \\
\hline $\mathbf{1 9}$ & $\begin{array}{l}\text { Aidfromrelieforganizations is } \\
\text { sufficient forour needsin general }\end{array}$ & $\mathbf{1 0 2 2}$ & $\mathbf{3 0 5 3}$ & $\mathbf{1 9}$ & Low \\
\hline $\mathbf{2 3}$ & $\begin{array}{l}\text { Theservicesprovided usour needs } \\
\text { somewhat }\end{array}$ & $\mathbf{1 0 1 5}$ & $\mathbf{3 0 1 0}$ & $\mathbf{2 3}$ & Low \\
\hline & Generalarithmeticmean & $\mathbf{2 0 5 1}$ & $\mathbf{3 0 1 5}$ & & High \\
\hline
\end{tabular}

Table(6)showsthatthemathematicalaveragesoftheproblemsrelatedtotheeconomicdimensionfacedby Syrianrefugeesrangedfrom (3.00to 1.15).The axisobtaineda totalarithmeticmean(2.51), ahigh level,andparagraphs(9and10)(0.00), whichisa highlevel.The paragraphs stipulate thathouseholdexpensesare morethan income.Our economicsituationinside thecampneedsconsiderablesupport. $\quad$ Thisisa greatproofofthepooreconomicsituationoftherefugeesinsidethecamp.

Thecamp.Somechildrenandwomenhavetoresorttobeggingforaliving, andthisbehaviorThefamily ispreparedtodoany work.Inthesecond place, paragraph(1) camewithanaverage of(2.98)anda standard deviation(0.19)fromthehighlevel.Ontheeconomicconditionsmakemy family'sfuture unstable). Thisindicatesthe refugee'sfearofthe economic conditionshemay face,whichhemay notbeabletocopewithduetothe severeweaknessof economicresources.Ontheotherhand,inthelast place,paragraph(20)camewithanaverage of(1.15)anda standard deviation(0.43).Itisfrom thelowlevel.Theparagraphstatesthatthe servicesprovidedtousmeetourneedssomewhat.Directservicesonlyand donotprovidesecondaryservices, especiallyinthe camp.

Resultsrelated to the fifth question: What are the problems related totheextension dimension facing the Syrian refugees?

Table (7) averagesandstandarddeviationsof the responsesof the studysample paragraphsabouttheaxis"problemsrelatedtotheguidingdimensionfacingSyrianrefugees" indescendingorder.

\begin{tabular}{|c|c|c|c|c|c|}
\hline $\mathrm{N} \#$ & Paragraph & $\begin{array}{l}\text { Arithmetic } \\
\text { average }\end{array}$ & $\begin{array}{l}\text { Standard } \\
\text { deviation }\end{array}$ & Ranking & Level \\
\hline 12 & $\begin{array}{l}\text { Theprograms provided to us in } \\
\text { psychological supportare inadequate. }\end{array}$ & 0.1 . & 2.32 & 1 & high \\
\hline 1. & $\begin{array}{l}\text { Theguidanceprograms } \\
\text { provided did nothelp us increaseself- } \\
\text { confidenceand senseof security }\end{array}$ & 0.18 & 2.8. & 0 & Average \\
\hline 10 & $\begin{array}{l}\text { Theguidanceprograms } \\
\text { provided did nothelp us } \\
\text { changethewrongbehaviors. }\end{array}$ & 0.11 & 2.80 & . & Average \\
\hline 13 & $\begin{array}{l}\text { Programs and activities inside } \\
\text { the camp do not meet our needs }\end{array}$ & 0.11 & 2.81 & 1 & Average \\
\hline 3 & $\begin{array}{l}\text { Programs and activities within } \\
\text { thecamp areprovided } \begin{array}{l}\text { inan } \\
\text { easylanguagethat } \\
\text { understands. }\end{array}\end{array}$ & 0.12 & 2.86 & 5 & Average \\
\hline 02 & $\begin{array}{l}\text { Theguidanceprograms } \\
\text { provided to us arenot useful in allareas }\end{array}$ & 0.12 & 2.38 & 8 & Average \\
\hline 12 & $\begin{array}{l}\text { Orientation programs donot } \\
\text { help us integrate and communicate } \\
\text { with others. }\end{array}$ & 0.22 & 2.83 & 3 & Average \\
\hline 16 & $\begin{array}{l}\text { Within the camp, weprovide } \\
\text { awareness programs in various fields. }\end{array}$ & 0.26 & 2.32 & 6 & Average \\
\hline 2 & $\begin{array}{l}\text { Ourorientation programs and } \\
\text { recreational activities help us adapt to } \\
\text { livingwithin the camp }\end{array}$ & 0.28 & 2.8 . & 2 & Average \\
\hline
\end{tabular}




\begin{tabular}{|c|l|c|c|c|c|}
\hline 11 & $\begin{array}{l}\text { We aretrained in the camp } \\
\text { through programs offered on how to } \\
\text { overcomefeelings of fearand anxiety }\end{array}$ & 1.23 & 2.82 & 12 & Average \\
\hline- & $\begin{array}{l}\text { Theguidingservices provided } \\
\text { tomebased on myneedsand } \\
\text { suitmydesires. }\end{array}$ & 1.25 & 2.3. & 11 & Average \\
\hline 1 & $\begin{array}{l}\text { The camp offers metraining } \\
\text { on how to deal with social and } \\
\text { emotional aspects. }\end{array}$ & 1.21 & 2.66 & 10 & Average \\
\hline
\end{tabular}

\begin{tabular}{|c|c|c|c|c|c|}
\hline 5 & $\begin{array}{l}\text { Offers us non-useful } \\
\text { orientation programs commensurate } \\
\text { with our capabilities. }\end{array}$ & 1.63 & 2.36 & 1. & Average \\
\hline 1 & $\begin{array}{l}\text { Ourtrainingand } \\
\text { implementation of the programs } \\
\text { provided to usin the camp } \\
\text { byspecialists with high abilityto } \\
\text { provideinformation easily. }\end{array}$ & 1.61 & 2.65 & 11 & Average \\
\hline 0 & $\begin{array}{l}\text { Theguidanceprovided within } \\
\text { thecamp helped medevelop } \\
\text { communication skills. }\end{array}$ & 1.33 & 2.6. & 15 & Average \\
\hline 6 & $\begin{array}{l}\text { Institutions provide } \\
\text { psychological support programs to us. }\end{array}$ & 1.88 & 2.30 & 18 & Low \\
\hline 18 & $\begin{array}{l}\text { Social workers listen to us } \\
\text { permanentlyinsidethe camp }\end{array}$ & 1.5 . & 2.88 & 13 & Low \\
\hline 8 & $\begin{array}{l}\text { Thespecialists in the camp are } \\
\text { always with usand their numberis } \\
\text { sufficient. }\end{array}$ & 1.12 & 2.81 & 16 & Low \\
\hline 15 & $\begin{array}{l}\text { Weparticipatein thedesign of } \\
\text { theguidanceprograms offered to us in } \\
\text { the camp. }\end{array}$ & $1 . .2$ & 2.86 & 12 & Low \\
\hline 11 & $\begin{array}{l}\text { Thesocial worker helpedme } \\
\text { get rid of someof thenegative } \\
\text { behaviors inside thecamp }\end{array}$ & $1 . .1$ & 2.52 & 02 & Low \\
\hline & General arithmetic mean & 1.22 & 2.16 & & Average \\
\hline
\end{tabular}

ItisclearfromTable(7)thatthearithmeticaveragesoftheproblems

relatedtotheextensiondimensionfacedby theSyrianrefugeesranged between 2.43and1.31.Theaxisobtaineda totalaveragemean(1.90), whichisfrom theintermediatelevel.Washigherthantheaverageofmy account. Itreached(2.43)andwithastandarddeviation(0.79), which is high.Theparagraphstatedthatthe programsprovidedtousinthefieldof psychosocial support are insufficient. This indicates thegreatweakness in thepsychologicalandsocialservicesprovidedtotherefugees. They have sufficientawarenessandknowledgeoftheexistenceoftheseservices, and inthesecondplacecameparagraph(13) onaverage(2.16)andstandard deviation (0.63)isofaveragelevel.Theparagraphstatesthattheguidance programs providedto ushave helpedus toincrease self-confidence and senseofsafety.Thisindicatesthatrefugeefamilieshavenotbenefitedfrom theawarenessprogramsprovidedwithinthecamp. Incontrast, inthe last place,paragraph(11)camewithanaverage of(1.31)anda standard deviation(0.59), whichisoflowlevel.Theparagraphstates:"Thesocial workerhelpedmegetridof somenegative behaviorsinside thecamp, Refugeesinthe primary andmainroleof thesocialworkeraspreviously reportedwithus.

\section{Resultsrelatedtothesixthquestion: Aretherestatistically significant differencesin theproblemsexperiencedbythe Syrian refugeesdue todemographic variables(gender,number offamily members)?}

OneWayANOVAwasusedanda testwasusedtoidentify the varianceinrefugee problemsattributedto thedemographic variables (gender andnumber offamilymembers). The resultsare asfollows: 
First: Gender:-

Table (8) T-testtoidentifythevariationinthelevelofproblems

\begin{tabular}{|c|c|c|c|c|c|c|c|}
\hline Source & Sex & $\begin{array}{l}\text { Arithmetic } \\
\text { average }\end{array}$ & $\begin{array}{l}\text { standard } \\
\text { deviation }\end{array}$ & Sample & $\begin{array}{l}\text { Degrees } \\
\text { of } \\
\text { freedom }\end{array}$ & $\begin{array}{c}\text { Value } \\
\text { OF } \\
\text { (T) }\end{array}$ & $\begin{array}{c}\text { Statistical } \\
\text { significance }\end{array}$ \\
\hline \multirow{2}{*}{$\begin{array}{l}\text { Psychological } \\
\text { problems }\end{array}$} & Male & 0.28 & 2.08 & 20 & \multirow[t]{2}{*}{126} & \multirow[t]{2}{*}{$-0.206-$} & \multirow[t]{2}{*}{$* 2.221$} \\
\hline & female & 0.05 & 2.00 & 16 & & & \\
\hline \multirow{2}{*}{$\begin{array}{c}\text { Family } \\
\text { problems }\end{array}$} & Male & 0.20 & 2.05 & 20 & \multirow[t]{2}{*}{126} & \multirow[t]{2}{*}{-1.808} & \multirow[t]{2}{*}{$* 2.222$} \\
\hline & female & $0 . .0$ & $2 . .1$ & 16 & & & \\
\hline \multirow{2}{*}{$\begin{array}{l}\text { Economic } \\
\text { problems }\end{array}$} & Male & 0.12 & 2.11 & 21 & \multirow[t]{2}{*}{126} & \multirow[t]{2}{*}{$-0 . .33-$} & \multirow[t]{2}{*}{$* 2.212$} \\
\hline & female & 0.56 & 2.13 & 16 & & & \\
\hline \multirow{2}{*}{$\begin{array}{l}\text { Indicative } \\
\text { problems }\end{array}$} & Male & 1.2. & 2.52 & 20 & \multirow[t]{2}{*}{126} & \multirow[t]{2}{*}{1.208} & \multirow[t]{2}{*}{2.253} \\
\hline & female & 1.82 & 2.12 & 16 & & & \\
\hline \multirow{2}{*}{$\begin{array}{l}\text { Problems asa } \\
\text { whole }\end{array}$} & Male & 0.1 . & 2.18 & 20 & \multirow[t]{2}{*}{126} & \multirow[t]{2}{*}{$-1.22 .-$} & \multirow[t]{2}{*}{2.28} \\
\hline & female & 0.01 & 2.18 & 16 & & & \\
\hline
\end{tabular}

experiencedbySyrianrefugeesdue tothegender variable.

Table(8)showsthattherearestatistically significantdifferencesin the levelof psychologicalproblemsandfamily problems, andthe

economicproblemsfacedbySyrianrefugees(2,377,respectively,4.626,2.928)(T)duetogendervariable.Thelevelofsignific ance(0.05)andless, andthe differencesin the arithmeticaveragesfor the three problemsin favoroffemales.Table(8)showsthattherearenostatistically differencesinthelevelofextensionproblems, andtheproblemsasawhole sufferedbytheSyrianrefugeesinthecamp1.903(respectively,1.926)(T) attributedtothesexvariable;Thesignificance level(0.05)andthe differences inthe arithmeticmean, ifany, did notreachthe levelof statisticalsignificance.

Second: Number of familymembers:-

TheOneWayANOVAtestwasusedtoidentifythevarianceinthe levelofproblemsexperiencedby Syrianrefugeesduetothevariable number offamilymembers.The followingtable (9) illustratesthis:Table (9):Singlevariancetesttoidentifythe variance inthe level of problemsexperiencedbythe Syrianrefugeesdue tothevariablenumber offamilymembers

\begin{tabular}{|c|c|c|c|c|c|c|}
\hline \multirow[t]{2}{*}{$\begin{array}{l}\text { Psychological } \\
\text { problems }\end{array}$} & $\begin{array}{l}\text { Betweengroups } \\
\text { Withingroups }\end{array}$ & $\begin{array}{c}\text { Total } \\
\text { squares } \\
18186 \\
78283\end{array}$ & $\begin{array}{c}\text { Degrees } \\
\text { of freedom } \\
3 \\
116\end{array}$ & $\begin{array}{c}\text { Average } \\
\text { squares } \\
18129 \\
18169\end{array}$ & $\begin{array}{c}\mathrm{F} \\
18415\end{array}$ & $\begin{array}{c}\text { Statistics } \\
18743\end{array}$ \\
\hline & TOTAL & 78369 & 119 & & & \\
\hline $\begin{array}{l}\text { Family } \\
\text { problems }\end{array}$ & $\begin{array}{c}\text { Betweengroups } \\
\text { Withingroups } \\
\text { Total } \\
\end{array}$ & $\begin{array}{l}18458 \\
88163 \\
88521 \\
\end{array}$ & $\begin{array}{c}3 \\
116 \\
119 \\
\end{array}$ & $\begin{array}{l}18153 \\
18176\end{array}$ & 28115 & 18118 \\
\hline \multirow{2}{*}{$\begin{array}{l}\text { Economic } \\
\text { problems }\end{array}$} & Betweengroups & 18179 & 3 & 18126 & 1818 & 18321 \\
\hline & $\begin{array}{c}\text { Withingroups } \\
\text { Total } \\
\text { Betweengroup }\end{array}$ & $\begin{array}{l}28343 \\
28422 \\
18992\end{array}$ & $\begin{array}{c}115 \\
113 \\
3\end{array}$ & $\begin{array}{l}18122 \\
18331\end{array}$ & 18479 & 18224 \\
\hline \multirow{2}{*}{$\begin{array}{l}\text { Indicative } \\
\text { problems }\end{array}$} & Withingroup & 238473 & 115 & 18224 & & \\
\hline & Total & 248466 & 118 & & & \\
\hline Problemsas & Betweengroups & 18111 & 3 & 18137 & 18519 & 18217 \\
\hline
\end{tabular}




\begin{tabular}{|l|c|l|l|l|l|l|}
\hline a whole & Withingroups & 28542 & 114 & 18124 & & \\
& Total & 28653 & 117 & & & \\
\hline
\end{tabular}

Table(9)showsthattherearenostatisticallysignificantdifferences inpsychologicalproblems, family problems,economicproblems, and extensionproblems.Theproblemsasawhole oftheSyrianrefugeesare attributedto thevariablenumberoffamilymembers, witha statistical valueof(1.509)(respectively)Notsignificantatthelevelof $(1.479,1.18,2.005,0.415)$ (F) significance (0.05) andless. Thisindicatesanagreement inthepointsofview amongthe sampleofthe study onthelevelof problemsexperiencedby theSyrianrefugeesaccordingtothe number of familymembers.

\section{Discussion of Results:-}

Discussionoftheresultsofthequestion:Whatare the problems relatedtothe psychologicaldimensionfacingthe Syrianrefugees?

Theresultsshowed that themostimportant problemsfaced by the refugeesweretheconcernoftheirchildren,whichmay facedifficult situations, asaresultofthedifficultconditionsthey liveinthecamp, followedby sufferingfrom neglectandmarginalizationby the internationalcommunity.Thisindicatesthatthe refugee believesthat the internationalcommunity isnotinterestedInhiscaseasarefugeedespite alltheassistancehe giveshim. Thisisevidentfrom thenextparagraph (livingunderconstantinstabilityandthreatofdeparture), whichledtotheir continuingdepression. Theresultsalsoshowedtheirlossof the problems theyfaceandtheirsenseofinsecurity, whichmakesthem feelveryafraid ofthefuture, Thisisconsistentwithsomeofthe previousstudies, which werecallfrom thestudy(Faraj,2008), whichindicatedthefeelingof refugeeinferiority andmarginalizationasaresultoflivingwithinthe camp.Thisresultisconsistentwiththecrisis theory. Itconsiders thatthe refugeefamily hasbeenexposedtoanaccidentorsituationthatis dangerousandis themainreasonfor thepsychologicalpressurethatthe patternundergoes. The situationortheincidentmay beone oraseriesof consecutiveincidents, Thisisduetothebeginningofthe Syriancrisisand the Syrians'oppositiontotheSyrianregime.This situationresultsin situationsofincreasingtensionandanxiety,especially whenthepattern failstoadapttothesituation. Thisistheresult of the use ofmethodsthat arenotusefultoconfrontit.ForwhatitproducesFromphysicalandmental disordertomooddisturbance, thinkingand doingunintendedacts, aswell aspainfulpreoccupationwiththe crisissituation.

Theresultsshowthatrefugeesdonotfeelshy whendealingwith others. Thisindicatesthattherefugeehasastrongpersonality andhasthe abilitytodealwithothersnaturally. The refugee crisishasnotaffectedhis relationswithothers. They haveanegativeattitudetowardstheirchildren andtreatthemwell,sotheytrytogetclosertoothersandtalktothem, and that'sa positive aspect.

Discussionoftheresultsrelatedtothe question:Whatare the problemsrelatedtothe familydimension, whichfacetheSyrianrefugees?

Theresultsshow thatoneofthemostimportantproblemsrelatedto thefamilydimensionconfrontingtheSyrianrefugeeswastheirfeelingthatthey wereunabletomeetthebasicneedsoftheirchildrenandthatthey wantedto escapetheirrealitybecauseofthe manyproblemstheysuffer as aresult oftheirconcernabout thechildren'seducation.Onverysmall grounds.Thisis similar totheresults of previousstudieswhich indicated thatthesocialproblemsfacingrefugeesare theeducationof children

Thatthe primaryeducationisavailable only inprivateschools, whichrequiresa lotofexpensescannotbe paidby therefugees, andthe resultsshowedthattheyfacemanyproblemswiththefamilyonverysmall grounds, whichleadstotheuseofscreamingthatmayworkwithchildren, methodofdisciplinechildrenWhentheymake mistakes, as aresultofthechildren'sfailure toobey the parents'directiveseasily.This resultisinlinewiththeresultsoftherefugeefamily lifeandthatfamily relations have beenaffected.The difficult livingconditions surrounding therefugeefamilyhavemadethem unabletomeettheirneedsandtheir abilitytoadapttothesituationThe newPafter resort.

Ontheotherhand, thelowestindicatorsofthefamily

experiencedbytheSyrianrefugeefamilyandtoalowdegree,Icannotact

problems decisions, andavoidmychildrentalkingtome abouttheirexternalrelations, If eelweak thinkingthatmyfamily couldhaveany people, andthisindicatesthatthesocialrelationsamongtherefugeesinside 
strong,andthattheyknoweachotherverymuch.

Discussionoftheresultsrelatedtothe question:Whatare the problemsrelatedtothe economic dimensionfacingthe Syrianrefugees?

Theresultsshowedthat themostimportant problemsrelatedtothe economicdimension of thecampare thatthe householdexpenditurewas greaterthan incomeandthattheeconomicsituationinsidethecamp requiresgreatsupportanddifficulty inprovidingtheirfamilies.Thejob opportunitiestha0t haveamaterialreturnontherefugees, whichleadsto the refugees notfeelingoptimistic abouttheir future economic situation, whichinturnlimitstheirpersonalaspirationsforabettertomorrow.Asthe situationof thematerialfamilystandsintheway ofachievingthe aspirationsoftheirchildrentoeducation.Becauseofthedifficulty of paying thetuitionexpensesoftheirchildren.Thismakesthem thinkalot aboutthe economicfuture of their childrenandtheirsense of optimism towardsa better future. One of the most importanteconomic problems facingtherefugeesinCairo, whichmadethemwithoutasteadyincomeandthereforewereunabletosatisfybasicneedsandpayt uitionfeesfor children.

Discussionofthefindingsonthequestion: What are the problemsrelated tothe extension dimension facingSyrian refugees?

Regardingtheresultsoftheextensionproblemsfacedby Syrian refugees, itwasfoundthatthereisa lack of provision of psychological supportandguidance programsforrefugees, aswellastheir lackof knowledge of the programsortheir places of delivery.Thisresult is consistentwithAbu Tarboush(2014)Psycho-socialimpact on childrento varyingdegrees, and thatthegreaterthechild'saccesstopsychosocial supportprograms, thegreaterhisability toexpress hispsychosocial problemslater, while the parentsindicatedthattheydidnotsee thechild's visit-orvisitthemasparentsforthatSoftwarehasSAItwastoalleviate theproblem whichtheysuffer,butthereisacategorywasfamiliarwith theseprograms, benefitingthemwell,especially inincreasingfeelingsof self-confidence, anda senseof security, andchange the wrongbehaviors.

Theresultsalso show thatthemost prominent the refugeefamilyandtheaverageextentoftheirfailuretobenefitfrom the guidance programsprovidedtothem;itdidnot helpthemtoincreaseself- confidenceandsense ofsecurity,andthat the guidance programsdidnot help themto change the misconduct within the camp, Programs and activitiesinsidethecampdidnotmeettheirneeds. Onthe other hand,the lowestindicatorsof theguidingproblemsexperiencedby therefugee family werelow, whichwereregularly attendedby socialworkersand withinthecamp, and thatthespecialistswerealwayspresentin thecamp andtheirnumberwassufficient. Thesocialworkerhelpsthemtogetridof

someofthenegativebehaviorsinsidethecamp.Thisisduetothefactthat therefugeesarenotfamiliarwiththeprimary andmainrole ofthe social worker as mentionedbefore.

\section{Results relatedtothequestion:Are the restatisticallysignificant differencesintheproblemsexperiencedbytheSyrianrefugees,dueto demographicvariables(gender, number of familymembers)?}

Theresultsshowedstatisticallysignificantdifferencesinthelevelof psychologicalproblemsandfamily problems. Theeconomicproblems favoroffemales, womensufferfrom experiencedbytheSyrianrefugeesareattributedtothegendervariable.In psychologicalandfamilyproblems asaresultofthemostimportantandmostimportantproblems.Fear,insecurity,frustrationandsurrender tothe bitterreality thattheyliveon theotherhand,wefindthatwomenare expectedtomaintainthecultureof societythroughtheirroleasa motherandwife, giventheconditionsoflife inapoorenvironmentwith narrow tents notWithadequatespacefor privacy,andwomen'svulnerabilitytoviolenceinsidethe camp.

\section{Study Recommendations:-}

Afterreviewingthe study results, thestudy reachedthefollowing suggestedrecommendations:

1. Worktoincrease the servicesprovidedtorefugeefamilies, which are workingtomeettheir needs.

2. Encouragingrefugeefamiliestoparticipatepositivelyinprograms providedbyrelevantorganizations.

3. Raisingawareness oftheroles of the socialworker,hisgoals, and thenature ofhiscareinthe organizations.

4. Organizingtrainingcoursesperiodically relatedtothecareof refugee families.

5. Activatetheroleofthemediatoraisetheissuesofrefugeefamilies andtheir problems.

6. Increasingtheroleof the governmentinraisingcommunity leadershipskillsoutside the camp,tomeettheneedsof asylumseekers. 
7. Activatetherole ofgovernmentinstitutionsinhighlighting the problemsofthefamiliesofSyrianrefugees,andlinkingthem tothe commitmentsandcommitmentsof donorcountries.

8. Linkingthecamptoacentralizedand databasethatdocumentsthedetailsofrefugeefamilies,monitorstheirmost

sophisticatedelectronic importantneeds, andhighlightstheirproblems.

\section{References:-}

1. AbdulRahim,TalaatHassan(1983),ThePsychologicalBasisof HumanGrowth,Cairo:ArabThoughtHouse.

2. AbuEl-Nasr,Medhat (2009),TheArtofSocialServicePractice,Cairo: Dar AlFajr PublishingandDistribution.

3. AbuTarboush,Ruba(2014), TheSocialandPsychologicalImpactsofthe SyrianCrisis onSyrianRefugee Childrenin Jordan,Unpublished Master Thesis, Universityof Jordan, Amman, Jordan.

4. Ali,MaherAbuAl-Maati(2009),GeneralPracticeinSocialWork,Cairo: HelwanUniversity.AlLawzi,Bassem,(2013)TheImpactofRefugeesontheEconomyoftheHostCountry,JordanCase Study..

5. Al-Wazzani,Khaled(2012),EconomicandSocialImpactsof theSyrian Crisis onthe JordanianEconomy,EconomicandSocialCouncil, Amman.

6. Al-Wazzani,Khaled(2014),TheEconomicandSocialImplications of theSyrianRefugeesonJordan,TheMughnim andMughram Framework,ConradAdenauer Foundation,JordanOffice.

7. Aqeel,HayaAbdullah(2004),Violence againstWomenduring Warand ArmedConflict,FieldStudy ofSyrianRefugeeWomenin Al- ZaatariCamp,UnpublishedMasterThesis,University ofJordan, Amman,Jordan.

8. Chatelard,Géraldine.2012.Jordan:ARefugeeHaven.MigrationPolicy.August, 2012. Via:http://goo.gl/xpThI

9. DalalAyham(2015), A Socio-economic Perspective on theUrbanisationof ZaatariCamp in Jordan, London.

10. Darwish,Yahia(1998), Glossary ofTermsofSocialService,Cairo: EgyptianInternationalPublishingCompany.

11. Derbas,Arig(2002),ProblemsofYouthinPalestinianRefugeeCampsinJordan, UniversityofJordan.

12. Faraj,AzzaAli(2008),RefugeeProblemsinCairo,ScientificConference21,Cairo:HelwanUniversity.

13. Fern,Hauck(2008),Immigrant Families Contemporary Society USA: TheVirginiaUniversity.

14. GabaryMohamed(1989),SocialWorkandFamily,ChildhoodandYouth

15. Care, Alexandria:The ModernUniversityOffice.Galabi,AliAbdelrazek(2013),SociologyandSocialProblems/New

16. VisionAlexandria University:Dar AlMaarifaUniversity.

17. Ibrahim,Qusay (2015),Theobstaclesfacingsocialcareservicesfor Palestinianrefugeesandwaysofaddressingthem fromthe perspectiveof internationalsocialservice, Jordanian Journal of SocialSciences,p.8,p.2, Amman, Universityof Jordan.

18. Luck,T.(2016).Jordan's SyrianRefugee EconomicGamble. Retrieved from http://www.mei.eduon(16-11-2017)

19. Mekdad,Faisal(2007),TheRealityofIraqiRefugeesinSyria,"ForcedMigration", $\quad$ No. 28, Universityof Oxford,England.

20. Phillips,Christopher.2012.TheimpactofSyrianrefugeesonTurkeyand Jordan.The WorldToday.Volume68,Number8/9.ChathamHouse. October2012.

21. Rashaida,Sahar(2014),Impactof City,Irbid,YarmoukUniversity,Jordan.

SyrianRefugeesonSocialSecurityinRamtha

22. Senhouryetal.(1991),SocialWorkinFamilyandChildhood,Cairo:DarAlHakim.

23. Turkey,BahaauddinKhalil(2015),Contemporary SocialProblems, Amman:Dar al-Masirah.

24. UNHCR.(2016a).SyriaRegionalRefugeeResponse.Retrievedfrom:http://data.unhcr.org/syrianon.

25. United NationsHigh CommissionerforRefugees (UNHCR) (2015), Za'tariCampinJordanmarksits thirdyearamidchallengesfacing the future ofthousandsof residents, Jordan.

26. UNRWA-UNHCR(2016),Zaatari,the largestArabrefugeecampsand thesecondworldwide.

27. Watenpaugh,K.D.,Fricke,A.L.\&King, J.R.(2014).Thewarfollows them:SyrianUniversityStudentsandScholarsinLebanon.Institute of InternationalEducation,Inc:US. 